\title{
Unknown Object Grasping Strategy Imitating Human Grasping Reflex for Anthropomorphic Robot Hand ${ }^{*}$
}

\author{
Tetsuya MOURI $^{* *}$ Haruhisa KAWASAKI** and Satoshi ITO** \\ ${ }^{* *}$ Faculty of Engineering, Gifu University, \\ 1-1 Yanagido, Gifu, 501-1193 Japan \\ E-mail: mouri@gifu-u.ac.jp
}

\begin{abstract}
This paper presents a strategy meant to allow anthropomorphic robot hands to grasp objects of unknown shape in imitation of the human grasping reflex. A 10-month-old baby may bend his/her thumb and four fingers in an attempt to grasp an object when it comes in contact with the palm. After grasping it, if the object is attempted to be removed from the baby's hand, the baby holds the object more strongly. In addition, the palm of the hand often makes initial contact with the object very lightly. This reaction is called the grasping reflex. In the proposed grasping strategy, each joint of the thumb and the fingers of a robot hand is controlled independently using the contact force affecting its adjacent fingertip side-link to imitate the grasping reflex. By setting a suitable contact force, the robot can grasp an object by both the fingertips as well as an enveloping grasp involving the palm at a uniform grasping force. Experimental results regarding the grasping of three-dimensional objects of unknown shape by an anthropomorphic robot hand called the Gifu Hand III are shown.
\end{abstract}

Key words: Anthropomorphic Robot Hand, Multi-Fingered Hand, Grasping of Unknown Object, Thumb Opposability, Tactile Sensor

\section{Introduction}

It is strongly expected that forthcoming humanoid robots will execute various complicated tasks based on communication with a human user. These humanoid robots will be equipped with anthropomorphic robot hands much like the human hand, and will eventually supplant human labor in the execution of intricate and dangerous tasks in areas such as manufacturing, space, the seabed and so on. It is expected that such robots will be able to grasp and manipulate objects whose shapes are not programmed into the robot.

A human can manipulate an unknown object using information obtained from a distributed tactile organ. Some studies have proposed a method to control the grasping of an unknown object using a tactile sensor or a force sensor based on an engineered approach $^{(1)-(6)}$. Endo ${ }^{(1)}$ proposed a method for controlling a grasping motion using local tactile information. Maekawa ${ }^{(2)}$ proposed a control method for dynamical grip strength while maintaining the stability of fingertip contacts. Konno ${ }^{(5)}$ considered grasping unknown objects by groping, while Maeno ${ }^{(7)}$ proposed a method for controlling grasping force modeled on the human grasping motion. However, these studies involve the robot hand grasping only a simple shaped object such as a sphere, rectangular prism and so on. Moreover, a sequence strategy from grasping an object to handling external forces has not been considered. One of the abilities essential for grasping and manipulating an object in 
humans is the grasping reflex, which appears during the postnatal period. This grasping reflex is considered an essential action for survival as a means of physically acquiring (a grasping action) and keeping (a withholding action) an object. It seems that the object is grasped with uniform contact force even if the object's geometrical form is unknown. It is considered that a robot hand having a grasping reflex should be able to easily grasp various unknown objects including soft objects ${ }^{(8)}$.

This paper presents a grasping strategy imitating the human grasping reflex as a means to allow the robot to grasp various unknown objects. The newly developed anthropomorphic robot hand, named the "Gifu hand III," which can realize dexterous object grasping and manipulation, is presented. An opposable thumb is proposed for grasping and manipulating the object, and a grasping strategy imitating the human grasping reflex is proposed. This grasping strategy involves three actions: a modifying action which adjusts the grasping position, a grasping action which ensures a positive grasp, and a withholding action which prevents the object's release. Experiments regarding the grasping of certain objects show that a dexterous grasp of an object can be realized using a simple control strategy.

\section{Anthropomorphic Robot Hand: Gifu Hand II}

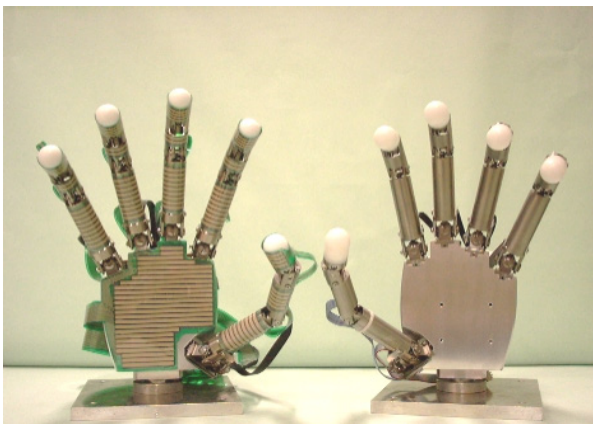

Fig.1 The developed Gifu Hand III

Table 1 Specifications of the Gifu Hand III

\begin{tabular}{|c|c|c|}
\hline \multirow{3}{*}{ Weight [kgf] } & Thumb & 0.25 \\
\hline & Fingers & 0.20 \\
\hline & Total & 1.40 \\
\hline \multirow{4}{*}{$\begin{array}{l}\text { Range of joint } \\
\text { motion [deg] }\end{array}$} & 1st joint & $\begin{array}{l}-28 \sim 28 \\
\text { (Thumb) } \\
-20 \sim 20 \\
\text { (Finger) }\end{array}$ \\
\hline & 2nd joint & $-10 \sim 90$ \\
\hline & 3rd joint & $-10 \sim 90$ \\
\hline & 4th joint & $-10 \sim 90$ \\
\hline \multirow{2}{*}{$\begin{array}{l}\text { Output force at } \\
\text { fingertip }[\mathrm{N}]\end{array}$} & Thumb & 2.8 \\
\hline & Fingers & 1.8 \\
\hline \multirow{4}{*}{$\begin{array}{l}\text { Output torque of } \\
\text { the thumb }[\mathrm{Nm}]\end{array}$} & 1st joint & 1.03 \\
\hline & 2nd joint & 0.58 \\
\hline & 3rd joint & 0.25 \\
\hline & 4th joint & 0.02 \\
\hline \multirow{4}{*}{$\begin{array}{l}\text { Gear ratio of the } \\
\text { thumb }\end{array}$} & 1st joint & 713.43:1 \\
\hline & 2nd joint & 384.69:1 \\
\hline & 3rd joint & 148.48:1 \\
\hline & 4th joint & 80.00:1 \\
\hline \multirow{4}{*}{$\begin{array}{l}\text { Band width of } \\
\text { the thumb }[\mathrm{Hz}]\end{array}$} & 1st joint & 10.4 \\
\hline & 2nd joint & 12.3 \\
\hline & 3rd joint & 7.4 \\
\hline & 4th joint & 9.5 \\
\hline
\end{tabular}

Recently, robot hands with built-in actuators ${ }^{(9)-(11)}$ have been developed. However, these hands present a problem in that their movement is unlike the human hand because of an insufficient number of fingers and finger joints. Our group developed the Gifu Hand I ${ }^{(12)}$ and the Gifu Hand II ${ }^{(13)}$. The Gifu Hand is a 5 -fingered hand driven by built-in servomotors, and has 20 joints with 16 DOF. To reduce the backlash in the gear transmission, which appears after long operation, the Gifu Hand underwent modification. This paper presents the improved robot hand called the Gifu Hand III. An overview of the Gifu hand III is shown in Fig. 1. The right and left hands are symmetrically designed and each has a thumb and four fingers. The design mechanisms of the thumb and fingers are shown in Fig. 2. The servomotors and joints are numbered from the palm to the fingertip. The thumb has 4 joints with 4 DOF and each of the fingers has 4 joints with 3 DOF. The movement of the first joint of the thumb and of the fingers allows adduction and abduction, and that of the second joint to the fourth joint allows anteflexion and retroflexion. Thus, the Gifu hand III has 20 joints with 16 DOF. Table 1 summarizes the 


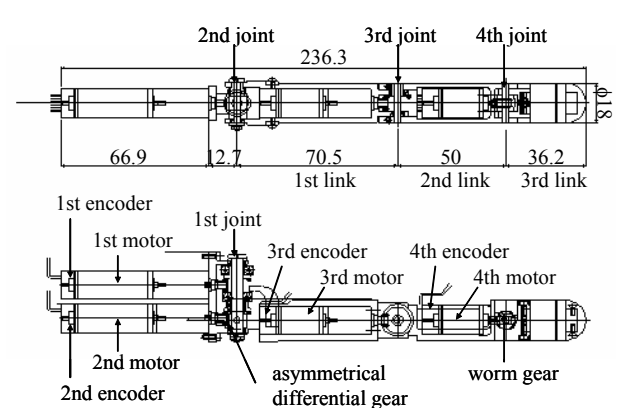

(a) Thumb
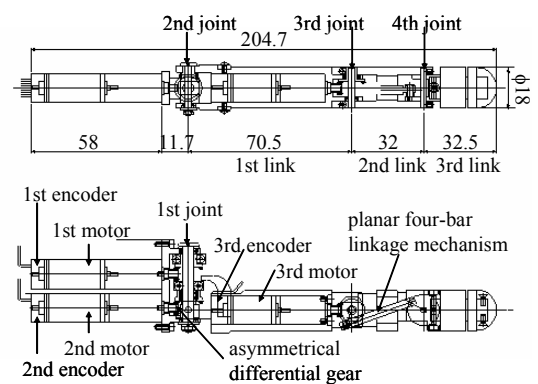

(b) Fingers

Fig.2 Mechanism of the thumb and the fingers

characteristics of the Gifu hand III. The design concept of the new robot hand depends on that of the Gifu Hand II.

The transmission structure is improved to decrease the joint backlash to less than 1.0 degree after long operation. The robot hand retroflexes to -10 degrees and has the same range of motion as the human hand ${ }^{(14)}$. Additionally, the joints' minimum bandwidth is $7.4 \mathrm{~Hz}$, while that of the human finger is, at most, $5.5 \mathrm{~Hz}$. This means that the robot hand can move more quickly than the human hand.

\subsection{Thumb Opposability}

The thumb of the human hand can move in opposition to all four fingers. The dexterity of the human hand in manipulating an object is based on thumb opposability ${ }^{(15)}$. The robot hand was designed to mimic the high degree of opposability of the human thumb.

To our knowledge, a method to evaluate thumb opposability has not yet been proposed. In order to evaluate it quantitatively, a performance index is defined by

$$
J=\frac{1}{d^{3}} \sum_{i=1}^{k} w_{i} v_{i}
$$

where $v_{i}$ denotes the volume of intersection between the mobility space of the thumb and that of the $i$-th finger, $k$ denotes the number of the finger except the thumb, $d$ is the longest length between the thumb and fingers, and $w_{i}$ is a weighting coefficient.

The thumb of the human hand plays an important role in grasping objects. The authors surmise that ranks of the digits contributing to grasping and manipulating objects ranges from the index finger to the little finger. Fingertip pinching forces between the thumb and the index, middle, ring, or little finger were measured. The subjects included 10 adult men. Accordingly, average and standard deviation in the case of the index finger was found to be $15.67 \mathrm{~N}$ and $3.76 \mathrm{~N}$, respectively. The ratios of the average of the fingertips' respective forces are: the index finger $=1.0$, the middle finger $=0.9$, the ring finger $=0.5$, and the little finger $=0.4$. The weighting coefficients, $w_{i}$, are proportional to these rations. The performance index of the Gifu Hand III $(J=0.0355)$, is 7.1 times better than that of the Gifu Hand II $(J=0.0050)$. The Gifu Hand III can grasp and manipulate an object over a broad range of fingertip positions.

\subsection{Planar Four-Bar Linkage Mechanism}

In the human hand, the fourth joint angle engages with the third joint angle almost linearly. In the Gifu Hand III, the fourth joint is driven by a planar four-bar linkage mechanism as shown in Fig. 3 (a). The relation between the third joint angle and the fourth joint angle is given as follows:

$$
l_{i}=\left[\left\{r_{i 3} S_{q_{i 30}+q_{i 3}}+r_{i 4} S_{q_{i 40}+q_{i 4}}\right\}^{2}+\left\{a_{i}+r_{i 3} C_{q_{i 30}+q_{i 3}}-r_{i 4} C_{q_{i 40}+q_{i 4}}\right\}^{2}\right]^{\frac{1}{2}},
$$

where $a_{i}$ is the length of the finger's second segment, $r_{i 3}$ and $r_{i 4}$ are the link radiuses 


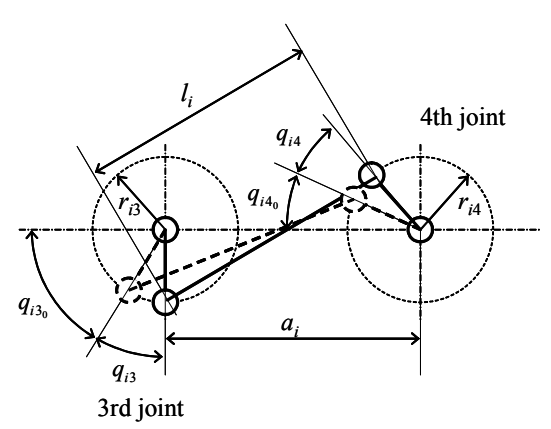

(a) Skeleton

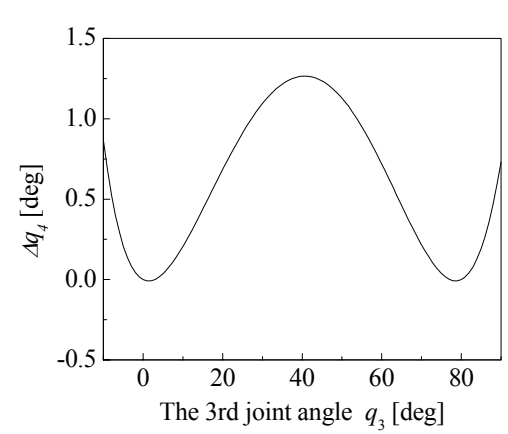

(b) Relation between the third and the fourth joints angles

Fig.3 Planar four-bar linkage mechanism

of the third and fourth joints, respectively, $q_{i 30}$ and $q_{i 40}$ are the initial angles of the planar four-bars linkage mechanism, $C_{q}=\cos q, S_{q}=\sin q, l_{i}$ is the length of the connection link, and the suffix $i$ refers to the $i$-th finger. The previous paper ${ }^{(12)}$ proposed a recursive numerical computation method for the fourth joint angle. However, that approach was time consuming. The present paper clarifies an explicit relation between the third joint angle and the fourth joint angle.

Equation (2) is satisfied for an arbitrary $q_{i 30}$ and $q_{i 40}$, because $l_{i}$ is a constant value. The fourth joint angle is written as follows:

$$
q_{i 4}=f_{i}\left(a_{i}, r_{i 3}, r_{i 4}, q_{i 30}, q_{i 40}, q_{i 3}\right)=\sin ^{-1}\left(\frac{\alpha_{i}}{\sqrt{\beta_{i}^{2}+\gamma_{i}^{2}}}\right)-\tan ^{-1}\left(\frac{\beta_{i}}{\gamma_{i}}\right)-q_{i 40},
$$

where

$$
\begin{aligned}
& \alpha_{i}=-2\left\{r_{i 4}\left(r_{i 3} C_{q_{i 30}+q_{i 30}}+a_{i} C_{q_{i 30}}\right)+a_{i} r_{i 3}\left(C_{q_{i 30}+q_{i 3}}-C_{q_{i 30}}\right)\right\}, \\
& \beta_{i}=-2 r_{i 4}\left(a_{i}+r_{i 3} C_{q_{i 30}+q_{i 3}}\right), \\
& \gamma_{i}=2 r_{i 3} r_{i 4} S_{q_{i 30}+q_{i 3}} .
\end{aligned}
$$

The explicit equation contributes to equation works to reduce the computation time of $q_{i 4}$. In the case of the Gifu Hand III, the parameters are set as follows: $a_{i}=32 \mathrm{~mm}$, $r_{i 3}=r_{i 4}=7 \mathrm{~mm}, q_{i 3_{0}}=70$ degrees, $q_{i 4_{0}}=30$ degrees. The ranges of motion of $q_{i 3}$ and $q_{i 4}$ are from -10 degrees to 90 degrees. Error $\Delta q_{i 4}$ is defined by

$$
\Delta q_{i 4}=q_{i 4}\left(a_{i}, r_{i 3}, r_{i 4}, q_{i 30}, q_{i 40}, q_{i 3}\right)-q_{i 3} .
$$

Figure 3(b) shows the calculations for $\Delta q_{i 4}$. The maximal difference is 1.27 degrees at $q_{i 3}=40.63$ degrees. This shows that the fourth joint of the robot finger can engage the third joint almost linearly in the manner of a human finger.

\subsection{Distributed Tactile Sensor}

Tactile sensors are mounted on the surfaces of the fingers and palm to pinch and envelop grasped objects. The tactile sensor is composed of grid-pattern electrodes and uses conductive ink in which the electric resistance changes in proportion to the pressure on the top and bottom of a thin film. The tactile sensor's insensitive area, i.e., a non-measuring area even if contact occurs, is $16.7 \%$ of the whole area, as described in Ref. (13). A distributed tactile sensor developed in cooperation with the Nitta Corporation for the Gifu Hand III is shown in Fig. 4. The characteristics of the tactile sensor are shown in Table 2. The electrode column width, the electrode row width, the column pitch, and the row pitch are modified dimensions of the Gifu Hand II. The insensitive area is increased to $59.1 \%$. Moreover, the numbers of detecting points on the palm, thumb, and each of the fingers are 313,126 , and 105 , respectively, with the total number of detecting points being 859 . As a 
result, the tactile sensor for the Gifu Hand III is expanded by 235 points relative to that of the Gifu Hand II. These improvements allow the Gifu Hand III to identify tactile information more accurately.

The effectiveness of the developed tactile sensor has been demonstrated experimentally. Figure 5 shows sequences of tactile output when an edge line of a rectangular solid is rotated on the palm equipped with the tactile sensor of Gifu Hand III overlapping that of Gifu Hand II. The average number of cell for detecting contact points for the Gifu Hand II and for the Gifu Hand III is 6.40 and 9.80, respectively, "cell" meaning the number of intersecting points between a row electrode and a column one. Because dimensions of each cell of the Gifu Hand III are smaller than those of the Gifu Hand II, it can more accurately detect contact points.

The fingertips can be fitted with 6-axis force sensors (BL. AUTOTEC Company), allow the robot to manipulate an object softly and control compliance for the robot hand's fingertips.

\section{Grasping Strategy Imitating Human Grasping Reflex}

This paper proposes a grasping strategy imitating the human grasping reflex in regard to an object with an unknown shape. A baby at about 10 months of age may bend his/her thumb and four fingers and grasp a rod when it is in contact with the palm. After grasping, if the rod is plucked from the baby's hand, the baby holds the rod more strongly. This reaction, as mentioned earlier, is called the grasping reflex. This grasping reflex consists of two actions ${ }^{(16)}$ :

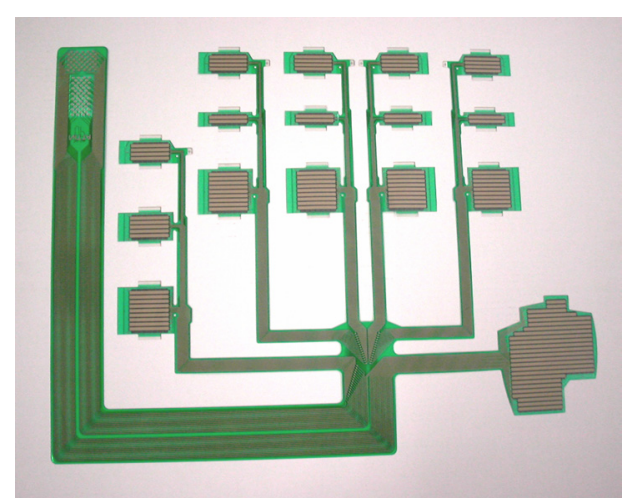

Fig.4 The developed distributed tactile sensor
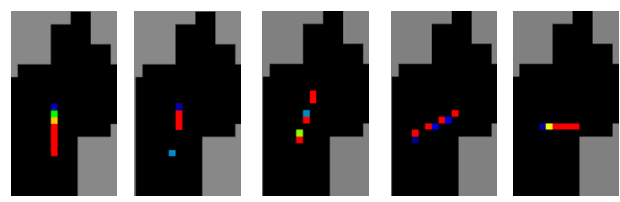

(a) Gifu Hand II

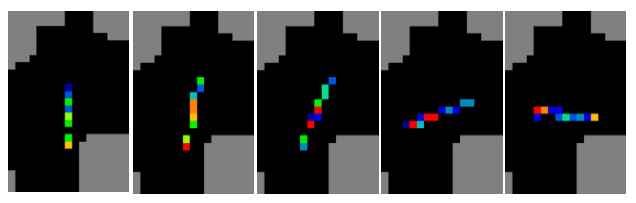

(b) Gifu Hand III

Fig.5 Output of the distributed tactile sensor

Table 2 Specifications of the distributed tactile sensor

\begin{tabular}{|c|c|}
\hline \\
\hline & 859 \\
\hline & 313 \\
\hline & 126 \\
\hline & 105 \\
\hline $\begin{array}{|ll|}\text { Number of detecting points } & \\
& \text { Total } \\
& \text { Palm } \\
& \text { Thumb } \\
& \text { Finger } \\
\text { Maximum load }\left[\mathrm{N} / \mathrm{m}^{2}\right] & \end{array}$ & $2.2 \times 10^{5}$ \\
\hline Electrode column width [mm] & 3.0 \\
\hline Electrode row width [mm] & 3.7 \\
\hline Column pitch [mm] & 4.0 \\
\hline Row pitch $[\mathrm{mm}]$ & 4.7 \\
\hline Sampling cycle [ms/flame] & 10 \\
\hline Resolution [bit] & 8 \\
\hline Thickness of sensor sheet [mm] & 0.2 \\
\hline Weight [gf] & 18 \\
\hline
\end{tabular}

1) Grasping action: generated when an object comes in contact with the hand.

2) Withholding action: generated when an object is plucked from the hand. In this section, the grasping strategy imitating these two actions will be considered.

\subsection{Modifying Action of Grasping Position}

Successfully grasping an object depends on the initial grasping position. In order to enhance the potential realization of stable grasping, the initial grasping position is modified 


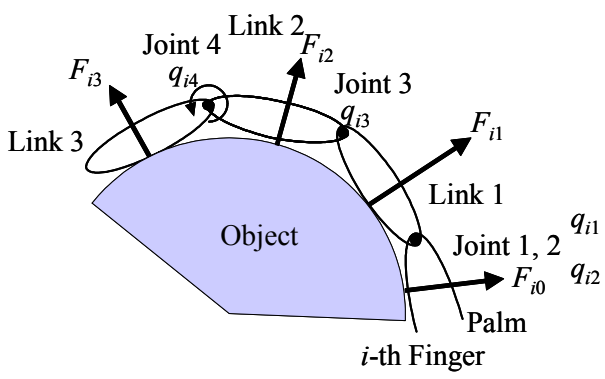

Fig.6 Links and contact forces

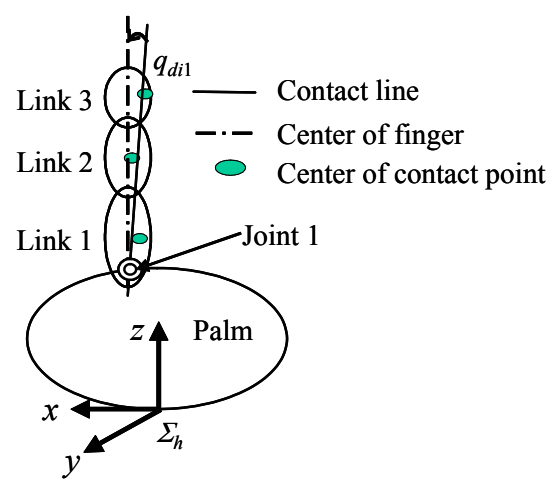

Fig.7 Desired first joint angle

by controlling the robot's arm's position. When an object comes in contact with the palm or fingers, the arm is moved by force control in a normal direction and is moved by position control in a tangential direction. The hybrid control moves the initial grasping position to the center of the palm. Here, the position of the grasped object is fixed in an external environment.

\subsection{Grasping Action}

Grasping an unknown-shaped object is made more secure by maximizing the number of contact points and enveloping the object. Moreover, it is expected that the object will be firmly grasped by the enveloping grasp. The proposed grasping strategy is outlined below.

\subsubsection{Anteflexion and Retroflexion}

When an object comes into contact with the hand, joints 2 to 4 of the thumb and the fingers are controlled independently to generate flexion such that the force of the side link of each joint of the adjacent fingertips equals a desired force. Figure 6 shows a contact state between the $i$-th finger and an object, where $q_{i j}$ and $\boldsymbol{F}_{i k}$ are, respectively, the angle of the $j$-th joint and the total contact force of the $k$-th link $(k=j-1)$ at the $i$-th finger. It seems reasonable that a velocity control be adopted at the non-contact state and a force control adopted at a contact state. Motor input $\tau_{i j}$, which drives the $j$-th joint of the $i$-th finger, is given by

$$
\tau_{i j}=-K_{V i j}\left(\dot{q}_{i j}-\dot{q}_{d i j}\right),
$$

where $K_{V i j}$ is the velocity feedback gain of the $j$-th joint of the $i$-th finger, and $\dot{q}_{d i j}$ is the desired velocity of the $j$-th joint of the $i$-th finger. Each desired joint velocity is set by velocity control in a non-contact state and force control in a contact state. The control performance depends on the contact points described in Ref. (8), because this method controls only the force feedback without information regarding the contact points. This paper proposes a control method using feedback torque equivalent to contact force as follows:

$$
\dot{q}_{d i j}= \begin{cases}\dot{q}_{c i j} & : \text { non - contact state } \\ -K_{f i j} J_{c i k}{ }^{T}\left(\boldsymbol{F}_{i k}-\boldsymbol{F}_{d i k}\right)-K_{f I i j} \int J_{c i k}{ }^{T}\left(\boldsymbol{F}_{i k}-\boldsymbol{F}_{d i k}\right) d t \quad \text { : contact state }\end{cases}
$$

where $K_{f i j}$ is the force feedback gain, $K_{f i j}$ is the force integral gain, and $\boldsymbol{F}_{d i k}$ is the desired force. $J_{c i k}$ is a kinematic Jacobian as follows:

$$
\dot{r}_{c i k}=J_{c i k} \dot{q}_{i j}
$$

where $\boldsymbol{r}_{c i k}$ is the contact point. The desired velocities on the hand's base side are set at larger values than on the fingertips' side. The link on the hand's base side generally makes first contact with the object. The robot hand can grasp the object at a constant contact force by setting the same desired forces. The fourth joint of each finger, excluding the thumb, cannot be driven directly because it is engaged with the third joint through the planar 


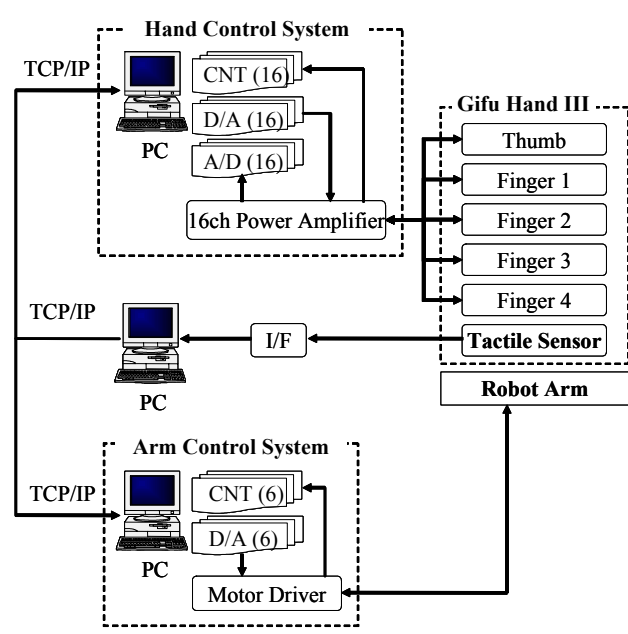

Fig. 8 The control system
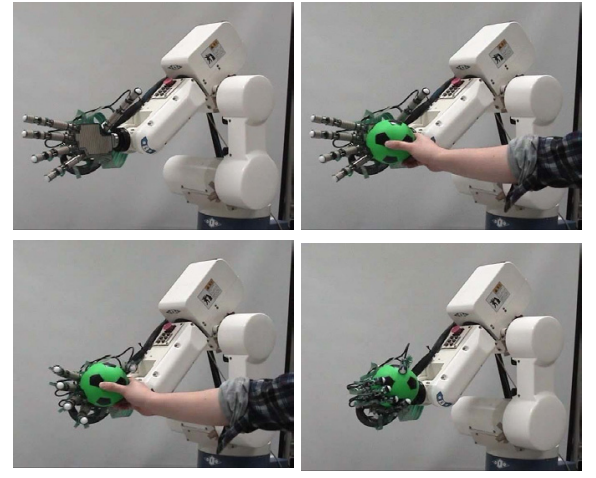

Fig.9 The process of spherical objects grasping

four-bar linkage mechanism. Hence, an instance where only either the third link or the fourth link is in contact with the object may occur. Therefore, the desired force of the third joint is obtained by adopting the largest of $\boldsymbol{F}_{i 2}$ and $\boldsymbol{F}_{i 3}$. This method controls the finger joints independently using tactile information. It is noted that pinching by the fingertips is possible by setting a suitable desired velocity $\dot{q}_{d i j}$.

\subsubsection{Adduction and Abduction}

The first joint is controlled to generate abduction and adduction according to the object's size. In order to realize this action, joint 1 of the thumb and each of the fingers is controlled by position and velocity control given by

$$
\tau_{i 1}=-K_{P i 1}\left(q_{i 1}-q_{d i 1}\right)-K_{V i 1} \dot{q}_{i 1},
$$

where $K_{P i 1}$ is the position feedback gain, $K_{V i 1}$ is the velocity feedback gain, and $q_{d i 1}$ is the desired joint angle. The desired joint angle is designed so that the center line on the surface of the thumb and each of the fingers coincides with the contact line, which runs from the axis of the first joint to the contact points using the least square method, as shown in Fig. 7. It is expected that the robot's grasp will more stable because the contact points come into line with a central line of the fingers.

\subsection{Withholding Action}

A withholding action occurs when the object is attempted to be removed from the hand. If an external force is applied to the grasped object, a constant grasping force cannot maintain its hold on the object. Therefore, a Euclidean norm of contact position velocity, $\left\|\boldsymbol{v}_{c}\right\|_{2}$, is added to the desired contact force, $\boldsymbol{F}_{\text {dik }}$, to maintain the grasping against the external force:

$$
\boldsymbol{F}_{d i k}=\left(1+K_{V C i k}\left\|\boldsymbol{v}_{c}\right\|_{2}\right) \boldsymbol{F}_{\text {dik_const }},
$$

where $K_{V C i k}$ is constant.

The three actions mentioned above constitute the proposed grasping strategy. The strategy is very simple, yet it can dexterously grasp objects of unknown shape because it does not depend on information regarding the shape of the object.

\section{Experiments}

\subsection{Experimental System}

Experiments demonstrate the effectiveness of the proposed grasping strategy using the 


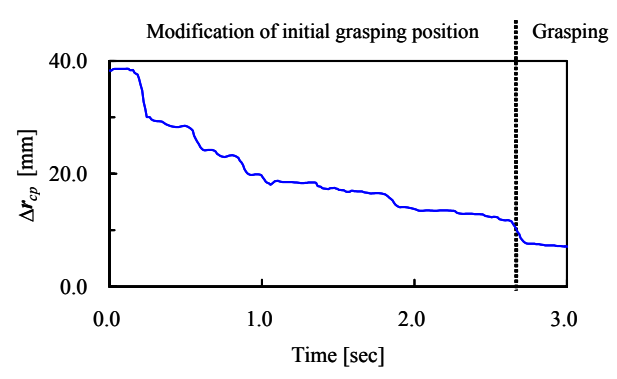

(a) Position error on the palm

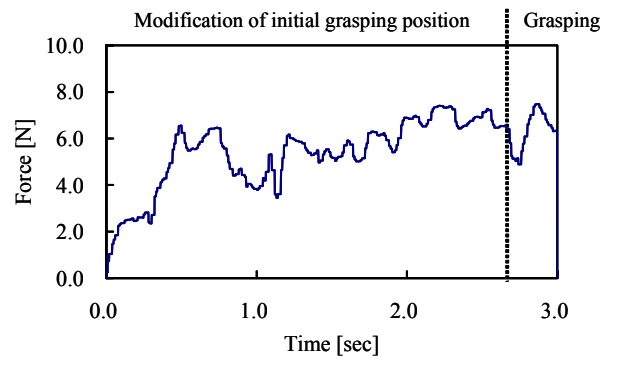

(b) Contact force on the palm

Fig.10 Modification of initial grasping position

Gifu Hand III. Figure 8 shows the developed PC-based control system for the experiments. The ART-Linux operating system is used for the robot hand, the 6-DOF robot arm (VS6354B, DENSO Company), and tactile sensor, this being a real-time operating system. The sampling cycles of the hand and arm controller are $1 \mathrm{~ms}$ and $2 \mathrm{~ms}$, respectively. The tactile sensor output is processed by a PC with a $10 \mathrm{~ms}$ period. The measured tactile data are transported to the hand and arm control PCs through a TCP/IP. The data sent from the distributed tactile sensor can easily be contaminated by noise since the circuit pattern is composed of electro rods. Therefore, tactile data are run through a low-pass filter having a cutoff frequency of $10.0 \mathrm{~Hz}$.

\subsection{Experimental Results}

Figure 9 shows the grasping sequence regarding a spherical object having a diameter of $110 \mathrm{~mm}$. The desired grasping position and contact force is $\boldsymbol{r}_{c p_{\_} d}=\left[\begin{array}{lll}-15.0 & 17.0 & 80.0\end{array}\right]^{T} \mathrm{~mm}$ and $0.3 \mathrm{~N}$, respectively. Transition from modifying action to grasping action occurs if it satisfies following equation:

$$
\Delta r_{c p} \leq 10.0 \text {, }
$$

where $\Delta r_{c p}=\left\|\boldsymbol{r}_{c p}-\boldsymbol{r}_{c p_{-} d}\right\|_{2}$ is the grasping position error. The grasping position on the palm and contact force are shown in Fig. 10. Both grasping position and contact force are close to the desired values. This grasping strategy allows the transition to grasping action.

Control of adduction and abduction affects the robot hand when grasping a small object or an object with a complicated shape. The experimental results of the hand grasping a sphere with a diameter of $90 \mathrm{~mm}$ are shown in Fig. 11. The results of control without adduction and abduction and with adduction and abduction are shown in Figs. 11(a) and (b), respectively. The robot grasps the object without dropping it in spite of varying the object's size. The grasping forces when adduction and abduction are controlled are show in Fig. 12. When the robot hand comes in contact with object, the desired velocity $\dot{q}_{d i j}$ changes in an almost stepwise manner and contact force is oscillatory. The contact forces converge to the desired force of $0.5 \mathrm{~N}$. The horizontal axis denotes the time from the beginning of the grasping action.

To demonstrate the effectiveness of the withholding action, the robot hand grasps a cylinder with a diameter of $80 \mathrm{~mm}$ and a mass of $101 \mathrm{~g}$. A weight with a mass of $302 \mathrm{~g}$ is dropped within the cylinder and slipping motion occurs. The features of the initial grasping state and withholding state are shown in Fig. 13. The velocity of the contact point, i.e., a variation of the centroidal point of all contact points, and contact forces (which are those of finger 1, finger 3, and the thumb) are shown in Fig. 14. Fingers 2 and 4 contact the object at non-measurable areas of the tactile sensor. Threshold of transition from a grasping action to withholding action is set at $10 \mathrm{~mm} / \mathrm{sec}$. This demonstrates the robot hand's success in grasping the object, and in increasing the contact velocity and contact forces.

Figure 15 shows various objects being grasped, these including a cylinder, a quadratic 


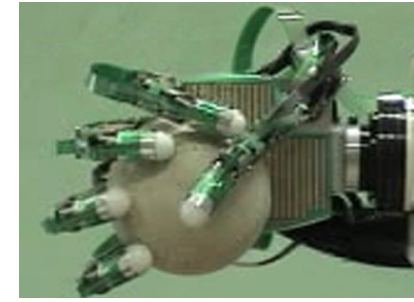

(a) Without adduction/abduction control

Fig.11 Effectiveness of adduction/abduction

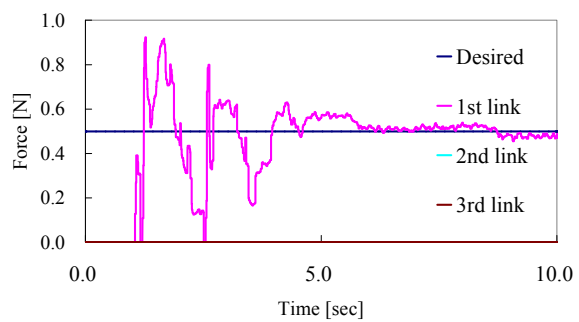

(a) Finger 1

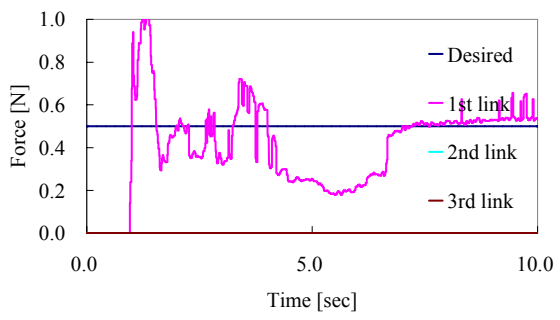

(c) Finger 3

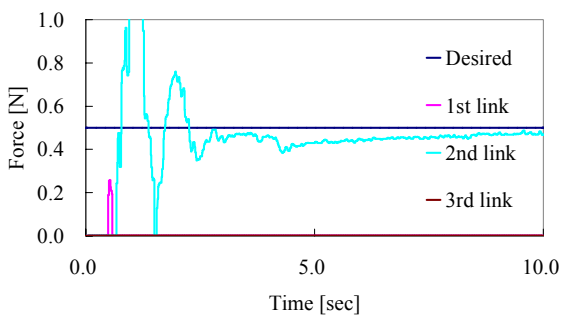

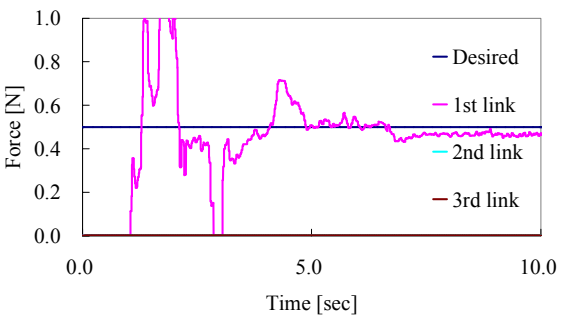

(b) Finger 2

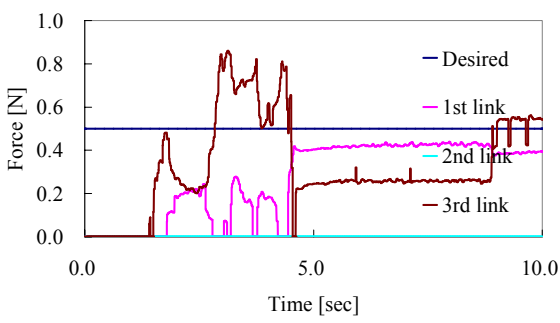

(d) Finger 4

(e) Thumb

Fig.12 Grasping force

prism, an object with a curved surface, and a human hand. The robot hand succeeds in grasping each of the objects in spite of their varying shapes and sizes.

The experimental results show that the proposed grasping reflex control is effective for unknown-shaped objects, as mentioned above.

\section{Conclusion}

This paper has considered providing a robot hand the ability to grasp objects of unknown shapes using tactile information. Grasping experiments are used to evaluate the performance of the newly developed anthropomorphic robot hand, the Gifu hand III. The robot's proposed opposable thumb, intended to play an essential role in the grasping and manipulating of an object, was evaluated. The distributed tactile sensor containing 859 detecting points can be attached to the surface of the robot's fingers and palm, allowing the 


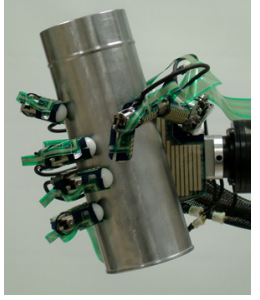

(a) Initial grasping state

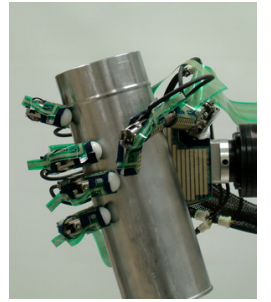

(b) Withholding state

Fig.13 Grasping at acting of external force

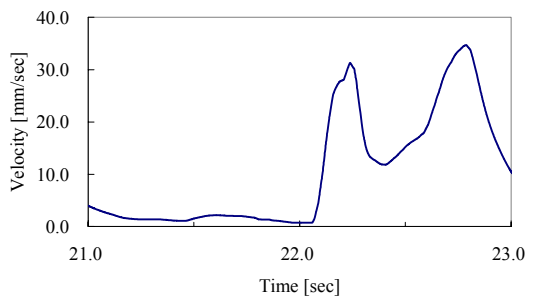

(a) Contact velocity

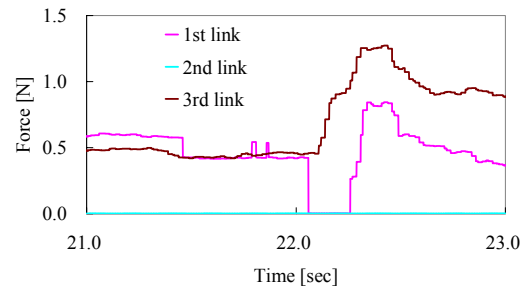

(c) Finger 3

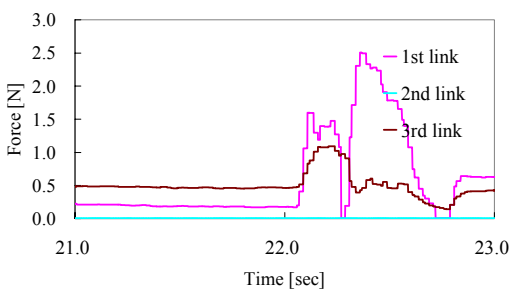

(b) Finger 1

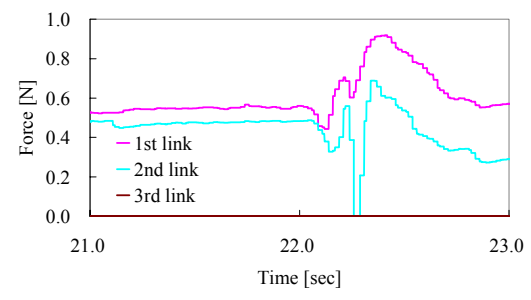

(d) Thumb

Fig.14 Grasping force

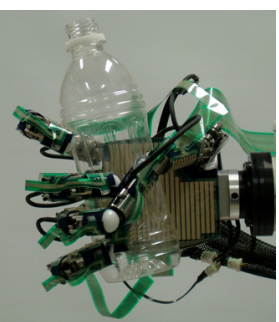

(a) Cylindrical object

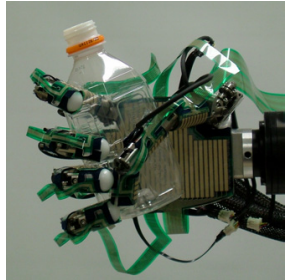

(b) Rectangular object

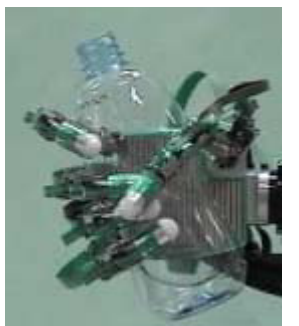

(c) Curved object

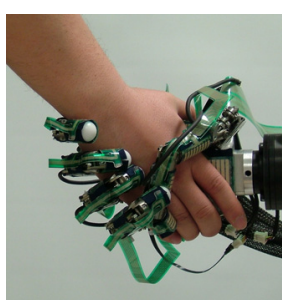

(d) Human hand

Fig.15 Grasping state of various objects

robot to dexterously grasp and manipulate objects. A grasping strategy imitating the human grasping reflex has been proposed, this grasping system being comprised of three actions: a modifying action which improves grasping position, a grasping action which ensures positive grasping, and a withholding action which prevents the release of the object. Experiments involving the grasping of certain objects showed that an object can be grasped dexterously though the use of a simple control strategy that does not depend on knowledge of an object's shape, thus allowing the robot hand to grasp objects whose shapes are unknown. This strategy can be applied to the care of elderly people and patients in welfare and medical care centers. 


\section{Acknowledgements}

The authors express their thanks to the partial funding and support from the Ministry of Education, Culture, Sports, Science and Technology (No. 14550443), and to the Gifu Robot Hand Group for its support.

\section{References}

(1) Endo, H., and Wada, M., Grasping Control Method of serial-Link Hand Based on Tactile Sense, Transactions of the Japan Society of Mechanical Engineers, Series C, Vol.59, No. 559 (1993), pp. 833-838 (in Japanese).

(2) Maekawa, H. et al, Dynamic Grasping Force Control Using Tactile Feedback for Grasp of Multifingered Hand, Proceedings of IEEE International Conference on Robotics and Automation (1996), pp. 2462-2469.

(3) Fischer, M. and Hirzinger, G., Fast Planning of Precision Grasp for 3D Objects, Proceedings of 1997 IEEE/RSJ International Conference on Intelligent Robots and Systems (1997), pp.120-126.

(4) Nakamura, Y., and Yamazaki, T., The Integration Theory of Reactive Behaviors and Its Application to Reactive Grasp by a Multi-Fingered Hand, Journal of the Robotics Society of Japan, Vol.15, No.3 (1997), pp.448-459 (in Japanese).

(5) Konno, A. et al, H, Development of a 3-Fingered Hand and Grasping Unknown Objects by Groping, Proceedings of the 1997 IEEE International Symposium on Assembly and Task Planning (1997), pp. 72 - 77.

(6) Tada, M. et al, Grasping of Unknown Objects Based on a Continuous Estimation of Relation between Finger Position and Contact Point, Proceedings of 17th Annual Conference of the Robotics Society of Japan, pp. 179-1180 (in Japanese).

(7) Maeno, T. et al, Control of Grasping Force by Estimating Stick/Slip Distribution at the Contact Interface of an Elastic Finger Having Curved Surface, Journal of the Robotics Society of Japan, Vol. 19, No. 1, pp.91-99 (in Japanese).

(8) Kawasaki, H. et al, Grasping of Unknown Object Imitating Human Grasping Reflex, CD-ROM of 15th IFAC Congress on Automatic Control (2002).

(9) Butterfass, J. et al., DLR-Hand II: Next Generation of a Dextrous Robot Hand, Proceedings of IEEE International Conference on Robotic and Automation (2001), pp.109-114.

(10) Carrozza, M. et al., Experimental Analysis of an Innovative Prosthetic Hand with Proprioceptive Sensors, Proceedings of IEEE International Conference on Robotics and Automation (2003), pp.2230-2235.

(11) Namiki, A. et al., Development of a High-speed Multifingered Hand System and Its Application to Catching, Proceedings of 2003 IEEE/RSJ International Conference on Intelligent Robots and Systems (2003), pp.2666-2671.

(12) Kawasaki, H., and Komatsu, T., Mechanism Design of Anthropomorphic Robot Hand: Gifu Hand I, Journal of Robotics and Mechatronics (1999), Vol. 11, No. 4, pp.269-273.

(13) Kawasaki, H. et al., Dexterous Anthropomorphic Robot Hand with Distributed Tactile Sensor: Gifu Hand II, IEEE/ASME Transaction on Mechatronics (2002), Vol. 7, No. 3, pp.296-303.

(14) Japanese Association of Rehabilitation Medicine ed., Display and Measurement of Range of Joint Motion, The Japanese Journal of Rehabilitation Medicine, Vol. 22, No. 2 (1974), pp. 127-132 (in Japanese).

(15) Suzuki. R., Brain and Hands, University Tokyo Press (1994), pp.6-8 (in Japanese).

(16) Hamajima, E., Reflex of Maturation Newborn Baby, The Medical journal of the Printing Bureau, Ministry of Finance, Japan, Vol. 33, No. 2 (1987), pp.25-36 (in Japanese). 